Maingebietes oder Frankreichs oder der Iberischen Halbinsel als „periglazial“ zu bezeichnen, wo sie doch ganz schlicht eiszeitlich, d. h. glazial sind. J. SCHÄFER allerdings ist der unverständlichen Meinung, die Bildung dieser Schotterterrassen falle „nach der bisher üblichen Auffassung sowieso ins Interglazial“ und nur die diluviale Terrassenbildung im Alpenlande sei vornehmlich in die (Früh-) Glazialzeiten zu stellen. Einer solchen falschen Vorstellung gegenüber ist jedoch immer wieder zu betonen, daß die pleistozänen Kaltzeiten das Primäre waren, mit weltweiten Wirkungen, zu denen in kalten Klimaten die Aufschotterungen in den Tälern und auch die Vereisungen gehörten. Es ist daher heute nicht mehr möglich, alle diese Erscheinungen allein aus der Enge alpiner oder perialpiner Schau betrachten zu wollen - wobei noch hinzuzufügen wäre, daß am südlichen Alpenrande weitgehend die gleichen Auffassungen bestehen, wie wir sie vertreten (vergl. S. VENZO: Revisione del Glaciale nella Bassa Val Cavallina. Atti della Società Italiana di Scienze Naturali 88 1949). Auf weiteres einzugehen, verbietet der Platzmangel.

R. Grahmann, 14.2.51

\title{
Plio-Pleistozängrenze und Günzeiszeit in Nordwestdeutschland
}

Von Daniel Wirtz und Henning I1lies in Hamburg. Mit 1 Abb.

I N H A L T :

Einleitung.

Sedimente und Sedimentationsbedingungen (ILLIES)

Glimmerton

Limonitsandstein

Feinsand

Kaolinsand

Einflüsse einer alten Landoberfläche.

Die Altersstellung der Schichtglieder des Morsumkliffs (WIRTZ)

Bisherige Deutungen

Neue Deutung.

Plio-Pleistozängrenze und Günzeiszeit.

Literaturverzeichnis.

\section{Einleitung}

Das Profil vom Morsumkliff auf Sylt ist das einzige in Nordwestdeutschland, das eine vollständige Schichtenfolge zwischen dem Glimmerton und der Mindel(=Elster) Moräne aufgeschlossen zeigt. Die Glieder dieser Folge wurden bisher in das Pliozän gestellt. Die Revision der Fauna des Sylter Limonitsandsteins (WIRTZ 1949) ergab, daß ein beträchtlicher Teil des Sylter Profils, in Übereinstimmung mit der Festlegung der Plio-Pleistozängrenze zu London 1948, dem ältesten Pleistozän (Günz) zuzurechnen sei.

Die Verfasser haben es daher unternommen, dieses Profil einer detaillierten sedimentpetrographischen und stratigraphischen Untersuchung zu unterziehen und damit ein Standardprofil für die Festlegung der Plio-Pleistozängrenze in Nordwestdeutschland zu geben.

\section{Sedimente und Sedimentationsbedingungen} (Von H. Illies)

G 1 i m m e r to n. Als Tiefstes des Profils am Morsumkliff (Abb. 1) tritt dunkler Glimmerton mit reicher Kalkschalerfauna auf. Der Ton weicht nur geringfügig von der üblichen Glimmertonfazies ab, wie sie kennzeichnend ist für das 
0

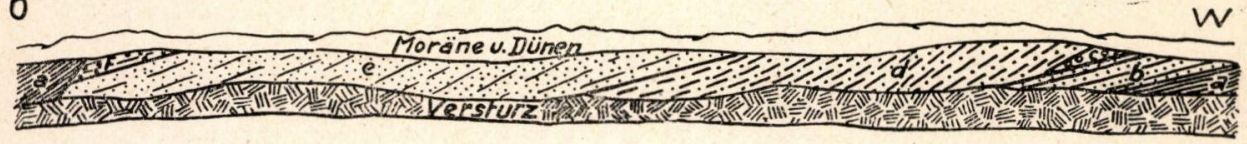

Abb. 1: Profil des Morsumkliff unterhalb des Gasthofes Nösse/Sylt. Länge $300 \mathrm{~m}$. $\mathrm{a}=$ Glimmerton, $\mathrm{b}=$ sandiger Glimmerton, $\mathrm{c}=$ Basiskonglomerat, $\mathrm{d}=$ Limonitsandstein, $\mathrm{e}=$ Feinsand, $\mathrm{f}=$ Kaolinsand. Die Schrägstellung sowie die Verwerfung im Ostteil des Profils sind durch glaziale Stauchung hervorgerufen.

gesamte jüngste Tertiär im östlichen Nordseebecken: einzelne Schill-Lagen lassen bereits den Einfluß schwacher Grundströmungen erkennen, und in den obersten Partien treten Mergelkonkretionen auf. Die obersten 10 Meter sind stark feinsandig und fossilfrei. Die Nordsee der Glimmertonzeit mit ihren ausschließlich feinklastischen Sedimenten bezeichnet ein stark ausgeglichenes Relief in den skandinavischen Liefergebieten.

Limonits andstein. Mit scharfer Grenze wird die tertiäre Schichtfolge vom Limonitsandstein überlagert, der stellenweise mit einem Basiskonglomerat, bestehend aus gerollten Molluskensteinkernen und groben Quarzgeröllen, einsetzt. Die Mächtigkeit des Limonitsandsteins beträgt 20 Meter. Petrographisch ist es ein fein- bis mittelkörniger Quarzsand mit limonitischem Bindemittel. In regelmäßigen Abständen von ca. 1 Meter tritt lagenweise Sideritsandstein auf, Die in Steinkernerhaltung bewahrten Mollusken sind vielfach pflasterartig angereichert. Fisch- und Knochenreste von Meeressäugern bilden stellenweise eine Art Bonebed und gaben zu verbreiteter Vivianitbildung Anlaß (WETZEL 1937). Das Gestein ist größtenteils von bogiger Kreuzschichtung mit Schichtneigungen bis zu $20^{\circ}$ durchsetzt. Die Sedimentation fand demzufolge in stark bewegtem Flachwasser statt. Nach HECK (1944) bildete das Meer damals eine den Nordwesten Schleswig-Holsteins umfassende Einbuchtung. Die vollständige Vererzung des Sediments läßt starke Eisenlösung auf dem benachbarten Festland vermuten, wie sie im kühl-humiden Bereich gegenwärtig im Gefolge flächenhafter Vermoorung auftritt.

Etwa 15 Meter über der Basis des Limonitsandsteins tritt ein zweiter Geröllhorizont auf. Unter den Geröllen überwiegen bis $5 \mathrm{~cm}$ große, stark abgerollte Milchquarze. Daneben treten an dieser Stelle zum ersten Mal die wenig gerundeten bis kantigen verkieselten Silurkalke zusammen mit frischen Granitund Feldspattrümmern auf (GRIPP 1922). Die Geröllführung ist petrographisch die gleiche wie sie später im Kaolinsand auftritt. Es fehlt dagegen die Korrosion der Feldspäte in den feldspatführenden Gesteinen. Die Gerölle weisen darauf hin, daß die Kaolinsandflüsse an der Schüttung des Limonitsandsteins bereits maßgeblich beteiligt waren. Stratigraphisch bedeutet das, daß während der Bildung des Limonitsandsteins weiter im Binnenland bereits fluviatile Aufschotterung nach Art des Kaolinsandes herrschte (vergl. hierüber die Untersuchungen von BERGER 1941 in Ostdeutschland).

Feins a nd. Durch Abnahme des Eisengehaltes leitet der Limonitsandstein allmählich zu der rund $35 \mathrm{~m}$ mächtigen Feinsandserie über. Es sind deutlich geschichtete fossilfreie Feinsande mit einzelnen tonigen Lagen und gelegentlich schwach angedeuteter Schrägschichtung. Ihre Zwischenstellung zwischen fluviatil beeinflußten Litoralsedimenten und echten Flußschottern läßt deltaischen Ursprung vermuten. In Delten größerer Ströme mit geringem Gefälle kommen häufig derartige Sedimente zum Absatz.

$\mathrm{K}$ a o linsand. Am Morsumkliff ist nur ein $5 \mathrm{~m}$ mächtiger Rest vor der glazialen Abscherung bewahrt geblieben. An zahlreichen anderen Stellen der 
Insel tritt der Kaolinsand dagegen ungestört an die Oberfläche und zeigt in Bohrungen Mächtigkeiten zwischen 20 und 30 Metern. Das Sediment hat seinen Namen nach dem Auftreten zahlreicher korrodierter und weißgebleichter Feldspattrümmer, die stellenweise zu Kaolin zersetzt sind. Die tiefsten Partien sind kreuzgeschichtet, darüber überwiegt regelmäßige Diagonalschichtung. Vielfach sind Geröllbänke und Tonflasern eingeschaltet. Meist sind es mittel- bis grobkörnige Sande mit seifenartig angereicherten Erzen, insbesondere Magnetit und Ilmenit. Die an sieben Aufschlüssen angestellte Analyse der Schrägschichtung (ILLIES 1949) läßt bei nördlicher bis nordwestlicher Fließrichtung den Einfluß eines breiten Stromsystems mit geringem Gefälle, sehr reicher Geschiebeführung und mittleren bis großen Fließgeschwindigkeiten erkennen. Bemerkenswert ist die 30 Meter erreichende flächenhafte A f s chotterung, die sich im gesamten Verbreitungsgebiet des Kaolinsandes größtenteils ohne nennenswerte vorhergehende Taleinschneidung vollzog. Ein großer Teil des norddeutschen Flachlandes von Ostdeutschland bis nach Ostfriesland und Südjütland wurde von dieser Aufschotterung betroffen, von der heute infolge der glazialen Ausräumung nur noch Reste vorhanden sind. Die Bewältigung derart erheblicher Schottermassen setzt ein wasserreiches Stromsystem mit starkem Gefälle im Einzugsgebiet und sehr geringem Gefälle im Unterlauf voraus. Die kräftige Heraushebung Skandinaviens gegenüber dem relativ stabilen norddeutschen Tiefland, dessen Relief zu dieser Zeit durch den mehr oder minder ebenen einstigen tertiären Meeresboden bestimmt wurde, ermöglichte bei gleichzeitiger starker Zunahme der Niederschlagsmenge eine flächenhafte Verlagerung der auf den skandinavischen Rumpfflächen angereicherten Restschotter nach Süden. Wie die an verschiedenen Stellen angestellten Schotteranalysen (STOLLEY 1900, HUCKE 1928) zeigen, folgten die Flüsse der Ostseesenke und durchflossen das norddeutsche Flachland in westlicher Richtung zur Nordsee, wo sie breite Delten aufbauten und dadurch die Nordseeküste wohl wesentlich vor die heutige Küstenlinie vorverlegten.

Die an den einzelnen Geröllhorizonten des Sylter Profils vorgenommene Schotteranalyse umreißt das Einzugsgebiet des Flußsystems. Wie im Limonitsandstein läßt sich auch im Kaolinsand eine scharfe Zweiteilung des Geröllbestandes in deutlich gerundete Quarze und wenig bis nicht gerundete Nichtquarze vornehmen. Um den Gegensatz zahlenmäßig ausdrücken zu können, wurde in den einzelnen Geröllhorizonten für jede Geröllklasse der durchschnittliche Rundungsgrad als Mittel von je 50 Messungen ermittelt. Der Rundungsgrad ist ausgedrückt als Quotient des Volumens einer Kugel mit dem Querschnitt gleich der Fläche des größten Hauptschnittes des Gerölls und des tatsächlichen Geröllvolumens. Der Wert beträgt für ein kugelförmig abgerolltes Geröll 1 und steigt mit abnehmender Rundung. Die Messungen erfolgten an Geröllen zwischen 14 und $34 \mathrm{~mm}$ Durchmesser.

Es wurden die folgenden Werte ermittelt:

$\begin{array}{lcc} & \text { Quarze } & \text { Nichtquarze } \\ \text { Kaolinsand } & 1,73 & 3,04 \\ \text { Limonitsandstein } & 1,75 & 2,29 \\ \text { Basiskonglomerat } & 1,54 & \text { fehlen }\end{array}$

Die $\mathrm{Qu}$ a r z e sind typische Gangquarze (Milchquarze), wie sie insbesondere für das kaledonische Skandinavien charakteristisch sind. Pegmatitquarze fehlen so gut wie vollständig. Die Gerölle besitzen eine eiförmige bis zylindrische (fluviatile) Abrollungsgestalt und erreichen im höheren Kaolinsand $16 \mathrm{~cm}$ Größe. Im Basiskonglomerat hat nachträgliche Brandungsabrollung stattgefunden (stärk- 
ste Geröllrundung). Das zahlreiche Auftreten solcher Quarze in Flußschottern, die ihren Weg durch die baltische Senke genommen haben, läßt in Skandinavien ein dem heutigen verwandtes Flußsystem vermuten. Das Zurücktreten fennoskandischen Kristallin-Materials in den Schottern ist wohl damit zu erklären, daß diese Gesteine damals noch nicht der Flußerosion unterlagen, da sie von der Sedimentdecke vollständig überlagert waren.

Die $\mathrm{Nich}$ tquarze des Limonitsandsteins und Kaolinsands sind größtenteils silifizierte Silurkalke. Ihre Oberfläche ist löcherig und kantig, vielfach auch zellig bis schlackig beschaffen. Im feineren Material verschärft sich noch der Gegensatz zwischen gerundetem und nicht gerundetem Material durch das Hinzutreten zahlreicher gebleichter Feldspattrümmer, die in scharfen Bruchstücken bis zu 20\% des Sediments ausmachen. Da auch die Härte dieser Komponenten geringer ist als die der Quarze, glaubte man die fehlende Abrollung nur dadurch erklären zu können, daß die Gerölle den rund $1000 \mathrm{~km}$ langen Transportweg durch eine Eisschollendrift überbrückt hatten (STOLLEY 1900, KOCH 1927, HUCKE 1928). Tatsächlich bezeichnet aber die zellig-schlackige Oberfläche mancher Silizifikate unvollständig verkieselte Kalke, aus denen der Kalk sekundär herausgelöst wurde. Der Sand an der Flußsohle tat ein weiteres, indem er der wechselnd starken Verkieselung folgend ein neues Relief modellierte. Die Eckigkeit der Feldspat-Trümmer ist dagegen lediglich durch die Spaltbarkeit bedingt. Während also bei den Quarzen der Sandschliff an der Flußsohle runde Flächen erzeugte, bewirkte er bei den Nichtquarzen das Gegenteil: eine Abnahme der Abrollung mit zunehmendem Transportweg.

Immerhin verdient zweierlei festgehalten zu werden: 1 . Die Transportenergie in dem gefällsarmen Unterlauf reichte aus, um von Zeit zu Zeit bis zu $15 \mathrm{~cm}$ große Gerölle zu befördern. $2.15 \mathrm{~cm}$ große Gerölle, rund $1000 \mathrm{~km}$ vom Einzugsgebiet entfernt, bedeuten unter Berücksichtigung des Abrollungsvorganges enorm große Anfangsgerölle und entsprechend hohe Fließgeschwindigkeiten im Oberlauf. Es muß daher weiter mit der Möglichkeit gerechnet werden, daß im skandinavischen Einzugsgebiet ein Teil des Transportweges durch Gletscherschub oder Eisschollendrift überbrückt wurde.

Während im feineren Material der Nichtquarz-Anteil 20\% erreicht, machen die größeren Gesteinsgerölle auf die Gesamt-Geröllführung nicht mehr als zwei Prozent aus. Neben ganz vereinzelten Graniten, Porphyren, Metamorphiten und präkambrischen Sandsteinen sind es größtenteils verkieselte kambrosilurische Kalke. An Hand ihrer reichen Fossilführung gelang es, sie auf ein ausgedehntes Heimatgebiet zwischen Estland und Mittelschweden zurückzuführen (STOLLEY 1900, 1929 und HUCKE 1928). Im höheren Kaolinsand treten vielfach verkieselte Wurzelböden vom Habitus der Braunkohlenquarzite hinzu (STOLLEY 1900, HUCKE 1928).

Die Kalke sind ausnahmslos silifiziert. Selbst aus $15 \mathrm{~cm}$ großen Silurblöcken ist aller Kalk durch Kieselsäure verdrängt. Die Verkieselung der Gerölle ist größtenteils im Heimatgebiet erfolgt. Dies beweisen Gelstrukturen und Diffusionsringe, die in keinerlei Richtungsbeziehung zưr heutigen Gerölloberfläche stehen. Man darf wohl mit THIELE (1941) annehmen, daß die Verkieselung bereits alttertiären Alters und als Verwitterungserscheinung auf der skandinavischen Landoberfläche erfolgt ist.

Einflüsse einer alten Landoberfläche. Während im Geröllhorizont des Limonitsandsteins völlig frische Feldspattrümmer auftreten und auch im tieferen Kaolinsand noch unzersetztes Feldspatmaterial vorkommt, sind im höheren Kaolinsand alle Feldspäte weiß gebleicht und gewöhnlich so stark 
korrodiert, daß sie bei einfacher Berührung zerfallen. Auch größere Kristallingerölle sind vielfach völlig zermürbt, also in einen Zustand versetzt, in dem sie nicht der geringsten mechanischen Beanspruchung durch einen Flußtransport mehr standhalten. Es hat demzufolge noch nach der Ablagerung des Kaolinsandes eine starke Korrosion des Mineralbestandes stattgefunden (KOCH 1927, HUCKE 1928, RICHTER 1935). Die Zunahme des Zersetzungsgrades zum Hangenden hin läßt erkennen, daß es sich um einen Verwitterungsvorgang gehandelt hat, der von der Kaolinsand-Oberfläche ausgegangen ist. Diese Annahme wird durch neuerliche Untersuchungen von WEYL (1949) bestätigt. Ein Schwermineral-Profil aus dem Kaolinsand von Lieth bei Elmshorn zeigte eine stark nach oben zunehmende Korrosion. In Zusammenhang mit diesem Prozeß dürfte die sekundäre rindenhafte Einkieselung mancher Kaolinsand-Gerölle zu erklären sein.

Eine starke Korrosion der Feldspäte im Verein mit einer vollständigen Herauslösung der Eisenverbindungen findet dort statt, wo zirkulierende humussaure Wässer längere Zeit auf ein Gestein einwirken können, ohne neutralisiert zu werden. Man kann annehmen, daß auf den kalkfreien Flußschottern bei Flußverlagerungen eine flächenhafte Vermoorung Platz griff, in deren Gefolge die Verwitterungserscheinungen eintraten. Zahlreiche Torf- und Braunkohlenflözchen, die an vielen Stellen im Kaolinsand erbohrt wurden, bestätigen diese Erklärung. Die Erscheinung weist auf das gleiche kühle und ungewöhnlich niederschlagsreiche Klima, welches bereits zur Deutung der Vererzung des Limonitsandsteins und zur Erklärung der flächenhaften Schüttung der Kaolinsande angenommen werden mußte.

Hiermit ist der altpleistozäne Sedimentationszyklus im Sylter Gebiet abgeschlossen. Der Kaolinsand wird gewöhnlich von einer älteren Mindel- (=Elster-) Moräne und einer jüngeren Riß-(=Saale-) Moräne überlagert. Die einzelnen ausgeschiedenen Stadien der Sedimentbildung im Sylter Raum sind zunächst weniger stratigraphisch oder klimatisch als durch den örtlichen morphologischen Wandel zu verstehen. Dennoch läßt sich ungezwungen ein Kulminationspunkt des pluvialen Klimazyklus erkennen, der im Sylter "Crag"-Profil im höheren Kaolinsand steckt. Dort erreichten $15 \mathrm{~cm}$ große, aus Mittelskandinavien stammende Gerölle das Gebiet der heutigen Nordseeküste, eine Transportenergie, die während der gesamten langen Abtragungsgeschichte des skandinavischen Rumpfgebirges sonst niemals erreicht wurde. Ob es während dieser Zeit in Skandinavien zu ersten Vereisungs-Ansätzen kam, ob die erwähnten Braunkohlenflözchen während jener Pluvialzeit oder in einem ersten „Interglazial“ entstanden, geht aus den sedimentpetrographischen Befunden nicht hervor.

Die Altersstellung der Schichtglieder des Morsum-Kliffs (Von D. Wirtz)

Bisherige Deutungen. Die Altersstellung der beiden fossilführenden Horizonte des Morsumkliffs ist schon frühzeitig erörtert worden. C. SEMPER (1856) erkannte als erster, daß die Fauna des Limonitsandsteins mit der des englischen Crags nahe verwandt sei, während die des Glimmertons mehr miozäne Züge besitze. In der Folgezeit geriet allerdings diese Erkenntnis wieder in Vergessenheit, und Glimmerton und Limonitsandstein wurden zusammen dem Obermiozän zugerechnet.

Erst GRIPP (1922) griff die Frage wieder auf. Er untersuchte die Fauna des Limonitsandsteins gesondert, unterstrich ihre Beziehung zur Crag-Fauna und 
stellte sie ins Unterpliozän. Den Glimmerton stellte GRIPP auf Grund eines dort gefundenen Zahnes von Hipparion gracile ins P o n t. Er schließt sich dabei der französischen Ansicht an, daß das Pont noch ins höchste Obermiozän gehöre. Der Limonitsandstein ist seiner Meinung nach etwas älter als die englische Lenham-Stufe.

STAESCHE (1930) befaßte sich im einzelnen mit dem Sylter Glimmerton. An Hand einer faunistischen Analyse horizontiert entnommener Proben zeigte er, daß der Sylter Glimmerton das jüngste Glied des nordwestdeutschen Glimmertons sei und daß auch innerhalb des Sylter Schichtstoßes noch eine fortschreitende Verarmung der miozänen Anreicherung borealer und pliozäner Molluskenarten vor sich ging.

THIELE (1941) diskutierte noch einmal die Altersfrage des Sylter Tertiärs, ohne neue Gesichtspunkte vorzubringen. Sein Versuch, auf Grund lithologischer Argumente die GRIPP'sche Gliederung anzufechten, erscheint wenig überzeugend.

Für den Kaolinsand gab es natürlich keinerlei Möglichkeit einer paläontologischen Altersbestimmung. Da er jedoch ohne irgendeine Zäsur aus der Feinsandstufe im Hangenden des marinen Limonitsandsteins hervorgeht, sah ihn GRIPP als fluviatiles Oberpliozän an und alle späteren Autoren (W. WETZEL, HECK und THIELE) sind ihm darin gefolgt.

So war das Sylter Profil bisher folgendermaßen altersmäßig eingestuft:

Kaolinsand

Limonitsandstein

Glimmerton
„Oberpliozän“

„Unterpliozän“

„Pont"
(= Schichtlücke unterhalb der LenhamStufe bzw. des Diestian)

(= obermiozäner mariner Horizont, der im belgisch-niederländischen Becken nicht mehr entwickelt ist).

$\mathrm{Ne}$ u e Deutung. Die Kenntnis der anglo-belgisch-niederländischen CragBildungen ist nun in neuerer Zeit erheblich verbessert worden. Gleichzeitig tauchte die Frage auf, wo in dieser Schichtfolge die Grenze zwischen Pliozän und Pleistozän zu ziehen sei. Dabei vollzog sich eine Verschiebung der Auffassungen über die Stratignaphie des „Pliozän“. Crag-Horizonte, die noch zur Zeit der GRIPP'schen Veröffentlichung als Oberpliozän galten, wurden nun als AltPleistozän angesehen. Anläßlich des Internationalen Geologen-Kongresses 1948 zu London wurde eine Kommission für die Plio-Pleistozängrenze eingesetzt. Sie entschied sich einmütig dafür, die marine italienische Folge als „Typen-Schichtfolge" zu betrachten und die pleistozäne Untergrenze an die Basis der Calabrischen Stufe zu verlegen. Sie erklärte, daß weiterhin in erster Linie marine Schichtfolgen für die Auffindung der Plio-Pleistozängrenze benutzt werden sollten.

Für die Crag-Bildungen der Nordsee wurde zwar keine endgültige Grenzziehung herbeigeführt, doch dominiert die Anschauung, daß zumindest die jüngere Hälfte des Red Crag bereits dem ältesten Pleistozän angehöre. Neuere, z. T. noch unveröffentlichte Untersuchungen über die Foraminiferenfaunen des belgisch-niederländischen Crag und die Bryozoen des anglo-belgischen Crag, von denen mir die Herren Dr. VAN VOORTHUYSEN und DRs.R.LAGAAIJ (Leiden) freundlichst Kenntnis gaben, verstärken die Wahrscheinlichkeit, daß die pliopleistozäne Grenze zwar nicht an der Basis des Red Crag aber doch in dessen tieferem Teil d.h. über der Walton-Stufe zu ziehen sei - wenn man den Empfehlungen der genannten Kommission für die Plio-Pleistozängrenze folgen will.

WIRTZ (1949) hatte nun die Fauna des Limonitsandsteins von Sylt einer Revision unterzogen und dabei gefunden, daß ihre Beziehungen zum englischen 
Crag doch enger seien, als GRIPP es angenommen hatte. Auf Grund der Fauna wurde der Limonitsandstein mit dem Waltonian des englischen Crag parallelisiert. Für die Einzelheiten der Beweisführung sei auf die genannte Arbeit verwiesen.

Durch diese Parallelisierung läßt sich die Schichtfolge des Morsum-Kliffs von Sylt zumindest an einer Stelle in das stratigraphische Schema von EnglandHolland-Belgien einhängen. Die Walton-Stufe und die gleich alte PoederlianStufe von Belgien-Holland zeigen in ihrer Fauna alle Anzeichen des Übergangs; gleichwohl muß man sie noch dem höchsten Oberpliozän zurechnen. Die gleiche Folgerung gilt nun auch für den Limonitsandstein, der also stratigraphisch den tiefsten Red Crag und das höchste Oberpliozän im östlichen Nordseebecken vertritt.

Die Frage des Alters des Sylter Glimmertons muß bis zum Abschluß laufender Arbeiten noch offen gelassen werden. Wie auch immer sie beantwortet werden mag: es läßt sich nicht leugnen, daß zwischen Glimmerton und Limonitsandstein ein Aufarbeitungshorizont liegt, der vielleicht mit einer Schichtlücke zusammenfällt. Außerdem sind die höchsten $10 \mathrm{~m}$ des Glimmertons fossilleer. So können wir über die paläogeographische und stratigraphische Bedeutung jenes Zeitraumes zwischen den letzten Fossilbänken des Glimmertons und den Küstensedimenten des Limonitsandsteins nichts Sicheres aussagen.

Wenn man also von einer Datierung der tiefsten Teile des Morsum-Kliffs zunächst absieht, so ergibt sich, daß die Plio-Pleistozängrenze unmittelbar im Hangenden des marinen Limonitsandsteins zu ziehen ist, wenn man stratigraphischen Prinzipien folgt. Würde man den Charakter der Sedimente zur Richtschnur der Gliederung machen, so müßte man den Limonitsandstein wohl bereits zum Pleistozän stellen. Die Glimmerton-Sedimentation läßt darauf schließen, daß in einer langen Zeit epirogener Ruhe und morphologischer Ausgeglichenheit nur wenig grobklastischer Detritus ins Meer geführt wurde. Das entspricht unseren Vorstellungen von der Zeit der mio-pliozänen Peneplanisierung. Der Wechsel am Ende dieser Phase erscheint in den Sedimenten der Nordsee zunächst langsam, später aber mit ruckartiger Schärfe abgebildet. Bereits im obersten fossilführenden Glimmerton finden sich Schill-Lagen, die reich an groben Quarzkörnern sind. Dann folgen die feinsandigen obersten $10 \mathrm{~m}$ fossilleeren Glimmer,,tons“. Und dann, nach einer Unterbrechung von unbekannter Dauer beginnt mit dem Limonitsandstein ein neuer Sedimentations-Zyklus, klimatisch und morphologisch bedingt, der in den höheren Kaolinsanden kulminiert und sich als ganzes schroff von dem älteren Zyklus abhebt. Er beginnt mit dem Auftauchen von gröberen Fluß-Sanden und Schotter-Bestandteilen in Küstensedimenten des obersten Pliozäns. Dann greift eine mächtige Schüttung von Delta-Sanden auf unser Gebiet über und macht schließlich reiner Fluß-Aufschotterung Platz.

Der Beginn dieses Zyklus konnte stratigraphisch mit der Basis des englischen Red Crag parallelisiert werden. Besteht die Ansicht von dem Ablauf der Entwicklung im Sylter Profil zu Recht und trifft die Altersdatierung seiner Basis zu, dann muß ein ähnlicher sedimentärer und klimatischer Ablauf auch in den gleichalten Serien Englands und Belgien-Hollands zu finden sein. Das ist tatsächlich der Fall. Der mit dem älteren Red Crag (Waltonian-Poederlien) beginnende Zyklus führt über den jüngeren Red Crag (Amstelien) zum Icenian, das den Höhepunkt des feuchtkühlen Klimas wie auch der Schuttzufuhr bezeichnet. Gleichzeitig verarmen die marinen Faunen progressiv, und auch die wenigen bisher bekannt gewordenen Braunkohlen weisen auf ein recht kühles Klima 
(THIERGART 1942, STOCKMANS 1943). Das Sylter Profil ist oberhalb des Limonitsandsteins fossilfrei, doch läßt der Vergleich mit dem englischen und belgisch-holländischen Profil den Schluß zu, daß der jüngere Red Crag und das Icenian in den Feinsanden und dem Kaolinsand enthalten sind. Das Maximum der fluviatilen Aktivität im höheren Kaolinsand dürfte mit dem Höhepunkt der Klimadepression im Icenian (englische Autoren zitiert bei ZEUNER 1945, SCHREUDER 1944, TAVERNIER 1943, ten DAM-REINHOLD 1941, VAN VOORTHUYSEN 1950) zusammenfallen. Eine Altersbestimmung des Sylter Zyklus im Einzelnen ist natürlich unmöglich, doch ist sein Beginn durch die Basis, seine Kulmination durch den oberen Kaolinsand angezeigt.

\section{Plio-Pleistozängrenze und Günzeiszeit}

Die erste der vier alpinen Eiszeiten, die Günzeiszeit, hat ein stark entwickeltes Gegenstück nur in der nordamerikanischen Nebraskan-Vereisung gehabt. Aus anderen Erdteilen sind die Zeugnisse spärlich und unsicher. Sicher ist dagegen, daß die Günzeiszeit weithin den Charakter einer pluvialen, kühlen Periode hatte. Die Untergrenze des Pleistozäns kann daher auch nicht durch glaziale Bildungen definiert werden. Die Kommission des Internationalen Geologenkongresses London 1948 für die Plio-Pleistozängrenze hat daher einmütig festgestellt, daß die Untergrenze des Pleistozäns dorthin zu verlegen sei, wo in der italienischen Neogenfolge die ersten Anzeichen einer Klimaverschlechterung zu erkennen seien. Das sei an der Untergrenze des Calabrien/Villafranchian der Fall. Wendet man diese Entscheidung „in order to eliminate existing ambiguities“ auf den englischen Crag an, so muß die Plio-Pleistozängrenze mindestens bis unter den Newbournian Crag hinabgezogen werden.

H. L. MOVIUS (1949) hat alles verfügbare Material zusammengestellt, aus dem die klimatisch-sedimentär-morphologische Wende der Plio-Pleistozängrenze auf der ganzen Erde ersichtlich wird. Es wird dabei verständlich, daß außerhalb der wenigen im ältesten Pleistozän vergletscherten Gebiete Umwelt und Leben vielfach noch ein Gepräge trugen, wie man es bisher wohl oft als "oberpliozän“ bezeichnete. Solche „oberpliozänen“ Ablagerungen werden dann von „eiszeitlichen" überlagert: dazwischen klafft ein scheinbarer Hiatus, der sich leicht überbrücken läßt, wenn man bedenkt, daß das Pleistozän nicht mit der „Eiszeit“ ident ist. Die Klärung der diesbezüglichen Begriffe durch die Beschlüsse des Londoner Kongresses ist deshalb sehr zu begrüßen: die Forderung, „that the plio-pleistocene boundary should be based on changes in marine faunas" eröffnet die Möglichkeit, bisher als pliozän betrachtete Horizonte dem Pleistozän einzugliedern und damit die „praeglazialen“ Lücken zu schließen.

Im Einklang mit diesem Prinzip kann man durchaus den Limonitsandstein von Sylt als Grenzschicht zwischen Plio- und Pleistozän betrachten. Er leitet den Sedimentationszyklus ein, für dessen Schichtglieder die Bezeichnung „Crag von Sylt" vorgeschlagen wurde (WIRTZ 1949). Es soll dadurch die Altersbeziehung zu den so ähnlichen und gleichaltrigen fluviatilen, deltaischen und marinen Bildungen Ostenglands, Belgiens, der Niederlande und Islands zum Ausdruck gebracht werden.

$\mathrm{Da}$ die entsprechenden Icenian-Ablagerungen Englands und der Niederlande im Großen gesehen der Günzeiszeit angehören, ist seit längerer Zeit erwiesen. Erwiesen ist auch, daß das Klima dieser Zeit zumindest kühl und feucht war. Dafür, daß es glazial im Sinne des späteren Pleistozäns gewesen sei, sprechen allerdings keine Anhaltspunkte. Zu glazigenen Bildungen der alpinen Günzvereisung gibt es von der Nordsee her keine Beziehungen. Die allgemeine Klima- 
depression scheint nun, wie im Vorstehenden gezeigt, auch in Skandinavien im höchsten Falle zu örtlichen Vergletscherungen besonders stark steigender Gebirgsteile geführt zu haben, die allerdings das Ausmaß der alpinen Günzvereisung nicht erreichten. Umso stärker war die Entwässerung der vom Atlantik her auf dieses steigende Land niedergehenden Niederschlagsmengen. Unter derartigen Verhältnissen sind die Cragschichten von Sylt entstanden. Sie vermitteln uns einen Eindruck von dem Geschehen im skandinavischen Raum, von dem auch im weiteren Umkreis Klima und Sedimentbildung der übrigen Nordseeküsten, beeinflußt waren.

ZEUNER (1945) glaubt, die von den Anhängern der Strahlungsgliederung angenommene Zweiteilung der alpinen Günzvereisung auch in England wiederzuerkennen und zwar in einer kalten Phase des jüngeren Red Crag und einer zweiten im höheren Icenian. Auch ZONNEVELD (1947) möchte eine solche Zweiteilung für das Icenian des Peel-Gebietes annehmen, wobei er die Tegelenstufe in das sogenannte Günz-Interstadial verlegt. Es ist nun vor allem fraglich, ob sich eine Zweiteilung der alpinen Günz durch Befunde im Gelände bestätigen läßt. Die Sylter Abfolge günzeiszeitlicher Sedimente läßt keinen Schluß auf eine derartige Zweiteilung zu, es sei denn, man würde die Geröllhorizonte im höheren Limonitsandstein und im höchsten Kaolinsand und ihr Fehlen in den Feinsanden in solcher Weise interpretieren. Aber der Sylter Crag ist ja im Wesentlichen ein Produkt von Gebirgshebung und Flußtätigkeit, im Unterlauf. Würde es da überhaupt möglich sein, eine „Interstadialzeit“ zu erkennen?

Auf eine weitere Schwierigkeit mag hier noch hingewiesen werden: die Frage der sogenannten „Donau-Vereisung“ B. EBERL's. Verf. hatte Gelegenheit, auf einer Exkursion anläßlich der Münchener Tagung der Deutschen Quartärvereinigung 1950 die wichtigsten Aufschlüsse zu besichtigen, die seinerzeit EBERL seiner Arbeit zugrundegelegt hatte. Es war wohl die vorwiegende Auffassung der Teilnehmer, daß das Beweismaterial jener Ablagerungen soweit sie tatsächlich glazigener Entstehung sind, nicht für eine Abtrennung vom Günz-Komplex ausreicht. Man gewinnt den Eindruck, daß der glazialen Akkumulation der Günzeiszeitlichen Gletscher eine Vorbereitungszeit mit periglazialer Sedimentation voranging, die weitgehend mit dem zusammenfällt, was bisher als „Donau-Vereisung" betrachtet wurde. Wenn dies richtig ist, dann stimmt es auch mit dem Befund von Sylt überein, nach dem die Kulmination des pluvialen Klimas am Ende des altpleistozänen Sedimentationszyklus liegt, ebenso wie die kühlste Phase des englischen Icenian den Ausgang jener Periode bildete. Man darf also zusammenfassend sagen: Zwischen der paläontologisch definierten Plio-Pleistozängrenze und dem Höhepunkt der Günz-Eiszeit liegt eine Übergangszeit, "die wahrscheinlich mit den alpinen „Donau-Vorstößen“ zu parallelisieren ist.

Es läßt sich auch noch nicht sicher sagen, wie lange Pluvialklima und Aufschotterung noch bis ins Günz-Mindelinterglazial hinein angedauert haben, ein Interglazial, das sich weiter westlich (Cromer Forest Beds) sehr deutlich von der vorhergegangenen Kaltzeit abhebt. Die von ILLIES beschriebenen Befunde lassen zwar eine zwischeneiszeitliche Landoberfläche über dem Crag erkennen, doch fehlen Ablagerungen, die man ihr zuordnen könnte - von geringen Ausnahmen abgesehen - in Nordwestdeutschland vorläufig ganz. So könnte man denken, daß die Aufschotterung noch einen Teil des Interglazials umfaßt hat. Fortschreitende Gebirgshebung und erneute Klimaverschlechterung führten dann rasch zu den glazialen Verhältnissen der Mindeleiszeit über, deren Moränenreste im Sylter Profil den Kaolinsand scharf schneiden. 
- Die nachfolgende Gliederungstabelle faßt den gegenwärtigen Stand der Kenntnis von den Altersbeziehungen der Schichtglieder über und unter der PlioPleistozängrenze im Nordseebecken zusammen.

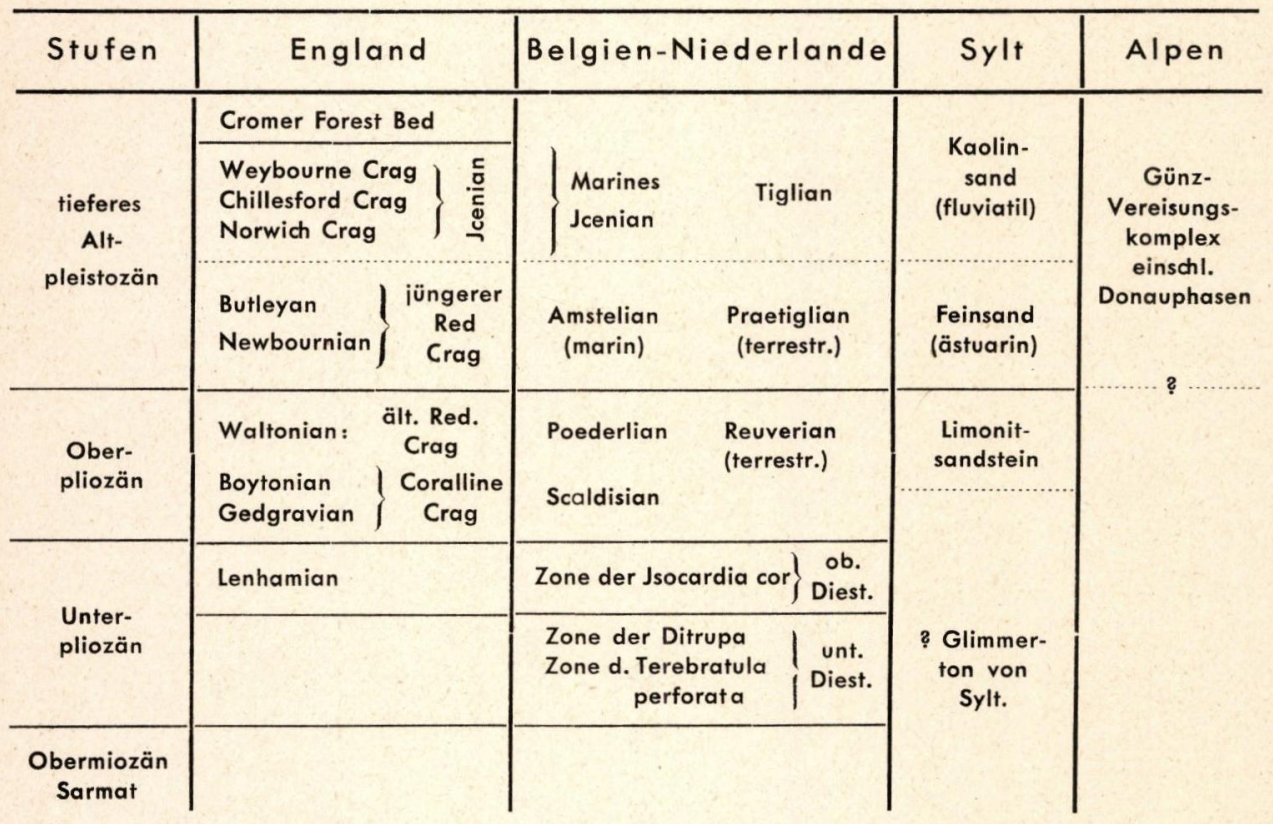

Literaturverzeichnis

BERGER, FR.: Kritisches zur Altersbestimmung des ostdeutschen Tertiärs. - Z. dtsch. geol. Ges. 93, S. 323 ff. 1941.

GRIPP, K.: Marines Pliozän und Hipparion gracile KAUP. Vom Morsumkliff auf Sylt. - Z. dtsch. geol. Ges. 74, S. 169-206, 1922.

HECK, H.-L.: Marines Pliozän in Schleswig-Holstein und der "Bredstedter Ton“. Jb. Reichsamt f. Bodenf. 63, S. 1-38, Berlin 1944.

HUCKE, K.: Neue Untersuchungen über das Pliozän in Pommern und Brandenburg. Z. f. Geschiebeforschg. 4, S. 157-184, 1928.

ILLIES, H.: Die Schrägschichtung in fluviatilen und litoralen Sedimenten, ihre Ursachen, Messung u. Auswertung. - Mitt. Geol. Staatsinst. Hamburg, Heft 19, 1949.

KOCH, E.: Beiträge zur Geologie des Untergrundes von Hamburg. - Mitt. Min. Geol. Staatsinst. Hamburg, 9, S. 1-109, 1927.

KRAUSE, P. G.: Das Pliozän Ostpreußens und seine Beziehungen zum nordwestdeutschen und westdeutschen Pliozän. - Abh. preuß. geol. Landesanst. N.F. 144, Berlin 1933.

MOVIUS, H. L. Jr.: Villafranchian Stratigraphy in Southern and Southwestern Europe. - Journ. Geol. Vol. 57, pp. 380-412.

OAKLEY, K. P.: International Geological Congress 18. Session, Proceedings of Section $\mathrm{H}$ : The Pliocene-Pleistocene Boundary. - Geol. Mag., 86, S. 18-21, London 1949.

PLEISTOCEEN - SYMPOSIUM: The stratigraphy of the pre-Riss Quaternary in the Netherlands. - Geologie en Mijnbouw, 12Jaargang Nieuwe Serie, pp.1-56, 1950.

RICHTER, KONR.: Zur Frage einer pliozänen Vereisung Norddeutschlands auf Grund neuer Funde bei Stettin. - Z. f. Geschiebeforschung, 11, S. 135-151, 1935.

SEMPER, J. O.: Paläontologische Notizen über den Sylter Limonitsandstein. - Kieler Schulzeitung für 1856 , S. $42-54$.

STAESCHE, K.: Zur Gliederung des obermiozänen Glimmertons. - Jb. preuß. geol. Landesanst. f. 1930, 51, Teil 1, S. 55-87, Berlin 1930. 
STOCKMANS, F.: Le lignite de Mol. - Bull. Mus. Roy. Hist. Nat., Brüssel 1943.

STOLLEY, E.: Geologische Mitteilungen von der Insel Sylt I. - Arch. Anthrop. Geol. Schlesw.-Holst. 4, S. 3-49, Kiel 1900. - Geologica varia von den Nordseeinseln. - 23. Jahresber. Niedersächs. Geol. Ver., S. 31-111, Hannover 1929.

TAVERNIER, R.: De kwartaire Afzettingen van Belgie. - Natuurwetensch. Tijdschrift, 25, S. 121-137, Gent 1943.

TEN DAM, A. und REINHOLD, TH.: Die stratigraphische Gliederung des niederländischen Plio-Pleistozäns nach Foraminiferen. - Meded. Geol. Stichting, Serie C, V, Nr. 1. Maastricht 1941.

THIELE, S.: Die Stratigraphie und Paläogeographie des Jungtertiärs in SchleswigHolstein. - N. Jahrb. Min. etc. Abt. B., Beil. Bd. 85, S. 1-143, 1941.

THIERGART, F.: Die Mikropalaeontologie als Pollenanalyse im Dienste der Braunkohlenforschung. - Schr. Brennstoffgeol., 13, Stuttgart 1940.

VAN VOORTHUYSEN, J. H.: The Plio-Pleistocene Boundary in the Netherlands based on the Ecology of Foraminifera. - Geologie en Mijnbouw, 12 Jaargang, pp. $26-31,1950$.

WETZEL, W.: Die Sedimenpetrographie des Sylter Tertiärs. - Schr. Naturw. Ver. Schleswig-Holst. 19, S. 204 -233, Kiel 1931. - Neue Beobachtungen am Jungtertiär von Sylt. - 28. Jahresber. niedersächs. geol. Ver., S. 74-104, Hann.1937.

WEYL, R.: Jungtertiäre Schlotten im Zechstein von Lieth bei Elmshorn (mit einer Bemerkung von H. ILLIES). - Schr. Naturw. Verein Schlesw.-Holst., 24, S. $74-81,1949$.

WIRTZ, D.: Die Fauna des Sylter Crag und ihre Stellung im Neogen der Nordsee. Mitteilg. Geol. Staatsinstitut Hamburg, Heft 19, 1949.

WOLDSTEDT, P.: Norddeutschland und angrenzende Gebiete im Eiszeitalter. 464 pp. Stuttgart 1950.

ZEUNER, FR.: The Pleistocene Period. - London 1945.

ZONNEVELD, J. I. S.: Het Kwartair van hat Peel-Gebied en de naaste Omgeving. Meded. Geol. Stichting, Serie C, VI, Nr. 3. Maastricht 1947.

Nachtrag während der Drucklegung

Seit Abschluß des Manuskriptes im Frühjahr 1949 haben P. THOMSON (N. Jb. Min., Abt. B., 1945-48, S. 367-369 und Geol. Jahrb., 65, S. 126), G. KREMP (Geol. Jahrb., 64, S. 511) und P. WOLDSTEDT (Z. dtsch. Geol. Ges. 100, S. 394, 1951) auf pollenanalytische Untersuchungen im Sylter Kaolinsand Bezug genommen. Alle diese Bemerkungen gründen sich indessen, was Sylt betrifft, auf Pollenanalysen THIERGART'S (1940) von Saprohumolithbänkchen im Kaolinsand des Roten Kliffs. Seine Untersuchungen ließen eine altpleistozäne Flora mit wenigen tertiären Relikten erkennen. Im Gegensatz zu WOLDSTEDT muß deshalb betont werden, daß kein grundsätzlicher Widerspruch zwischen der pollenanalytischen und der unsrigen Auffassung vom Alter des Sylter Kaolinsandes besteht, wenn man die Einstufung des Sylter Limonitsandsteins ins höchste Oberpliozän zugrunde legt.

\title{
Untersuchungen an nordwestdeutschen Interglazialen
}

\author{
Von P. Woldstedt, U. Rein und W. Selle. Mit 4 Abb.
}

\section{Vorkommen und Lagerung (P. WOLDSTEDT)}

Bei meinen langjährigen geologischen Aufnahmen in Nordwestdeutschland erhob sich immer wieder die Frage der Gliederung des dortigen Pleistozäns und insbesondere die Frage der Einordnung der Interglaziale. Über das Alter der verschiedenen Interglazialbildungen Nordwestdeutschlands ist auch früher schon viel diskutiert worden. Ich erinnere an die lebhaften Erörterungen über das Alter der Lauenburger Torfvorkommen. Eine erste pollenanalytische Unter- 